Chapter 20

\title{
Metal-Based Therapeutics for Leishmaniasis
}

\author{
Ana B. Caballero, Juan M. Salas and \\ Manuel Sánchez-Moreno \\ Additional information is available at the end of the chapter \\ http://dx.doi.org/10.5772/57376
}

\section{Introduction}

\subsection{Metal-based drugs and their growing application to the treatment of parasitic diseases}

When we speak of metals in medicine, many of us still associate them almost unconsciously with toxic rather than curative effects. However, despite the known toxic effect of some metal ions in humans, many metal ions (in adequate dosages) are required for many critical functions in our organism. Scarcity of some of them even can lead to a disease. Well-known examples include anemia resulting from iron deficiency, growth retardation arising from insufficient zinc, and heart disease in children owing to copper deficiency.

Metals have been used for medicinal purposes since ancient times. The earliest evidence of their therapeutic application has been dated back to 1500BC in Ebers Papyrus, Egypt. Among 700 magical formulas and remedies, this ancient manuscript describes the use of copper to reduce inflammation and the use of iron to treat anemia. Later on, the alchemical practice in the Middle Age made a significant use of metals like gold or arsenic to prepare medicinal compounds and elixirs. In the $16^{\text {th }}$ century, antimony was introduced by Paracelsus as a general panacea and was considered as one of the Seven Wonders of the World.

In the early $20^{\text {th }}$ century, the physician Paul Elhrich (Nobel Prize 1908) discovered an impresive therapeutic effect of the compound arsenophenylglycine to treat sleeping sickness (Trypanosoma disease) and developed the first effective medicinal treatment for syphilis, also arseniumcontaining drug Arshphenamine, which was commercialized under the name Salvarsan. The concept of chemotherapy was born. At this time, other metallodrugs appeared. Sodium vanadate and derivatives of bismaltolato oxovanadium(IV) complexes started to be applied to lower levels of blood sugar in diabetic patients, and gold(I) complexes such as Auranofin, sodium aurothiomalate and aurothioglucose were prescribed to treat rheumatoid arthritis 
(Figure 1). In 1987, sodium aurothiomalate was also used to treat 10 patients with kala-azar and showed an excellent clinical response.[1]

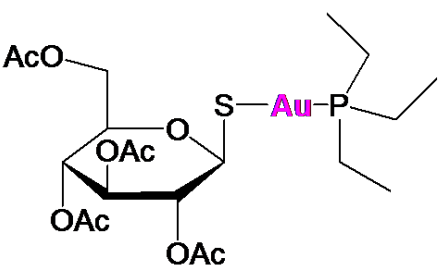

Auranofin
(Ridaura $^{\circledR}$ )

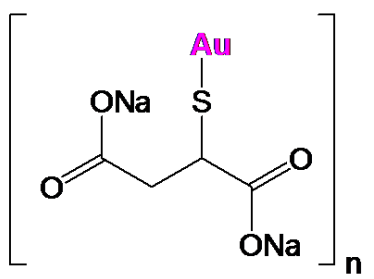

Sodium aurothiomalate
(Myocrisin $\left.{ }^{\circledast}\right)$

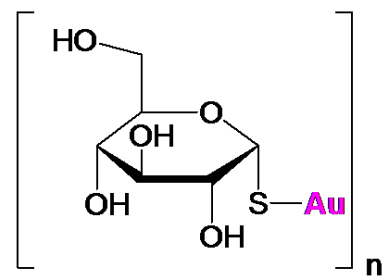

Aurothioglucose

Figure 1. Gold-based drugs most commonly used for the treatment of rheumatoid arthritis. Sodium aurothiomalate has also shown chemotherapeutic effect against kala-azar.

Despite these early demonstrations of the potential of metals to treat diseases, organic drugs have traditionally dominated modern medicinal chemistry and pharmacology. It was the serendipitous discovery in 1969 of the anticancer properties of cisplatin, a Pt(II) complex, which propelled dramatically the research on metal ions in modern medicine until nowadays, not only in therapy but also in diagnosis. Examples of the latter are radiolabeling of compounds with ${ }^{99 \mathrm{~m}} \mathrm{Tc}$ for X-ray imaging and use of Gd(III) complexes as MRI agents. Moreover, this event marked the change from an empirical discovery into a rational design of new metallodrugs and the consequent development of medicinal inorganic chemistry as a mature research discipline.

The increasing interest in the research of metal compounds with potential applications in medicine along the last decades has come along to a deeper understanding of the reactivity of metal ions and their interaction with a wide range of biomolecules such as DNA and proteins. [2] Scientific community has realized that either coordination or organometallic chemistry offer wide possibilities to develop novel metal-based drugs bearing quite different mechanisms of action aiming at different targets.

The dramatic incidence and economic impact of cancer diseases in modern world and especially in developed countries has led research on medicinal inorganic chemistry (and still is) to focus mainly on development of antitumoral compounds of different metals. This includes their design to specifically attack cancer cells and interact directly with DNA, with protein active sites or with smaller biomolecules of key importance in cancer development, as well as improving their biodistribution. As a result, a number of metal complexes with antitumoral potential have been developed in the last years, mostly of platinum and ruthenium, and some of them have provided excellent results. In fact, drugs like oxaliplatin are currently used to treat colorrectal cancer. Moreover, the antimetastatic drug NAMI-A is under the last phase of clinical evaluation. 
On the other hand, a comparatively smaller progress has been made in the discovery of new metal compounds to treat tropical parasitic diseases, which has been mostly based on an empirical use. Various inorganic salts have been administered against the major tropical diseases, sometimes with very good results.

The best-known example is a series of antimony compounds such as sodium stibogluconate (Pentostam) and meglumine antimoniate (Glucantime). These compounds were developed more than 60 years ago and still constitute the treatment of choice for some forms of leishmaniasis. However, antimonial-based treatments usually present toxicity problems, limited efficacy and emerging resistance. This leads scientists to explore other metal ions in search of improved therapies. In addition no structure-function correlation studies have yet been performed on antiparasitic metal-based compounds. These arguments open the way to new mechanistic investigations in this research area for optimization of the identified metal leads and development of new ones.

But what can metals offer towards improved antiparasitic therapies? Metal ions offer a wide range of coordination numbers and geometries, redox states, and thermodynamic and kinetic characteristics. This, along with the possibility to rationally combine the intrinsic properties of a metal ion with a bioactive ligand/s bearing therapeutic interest, provides innumerable possibilities for drug design and an extremely wide spectrum of therapeutic activity not readily available to organic compounds.

One of the most used design approaches is grounded in the metal-drug synergism that results from the attachment of a metal moiety to the structure of an organic drug. [3] This synergy gives rise to two main effects:

a. An enhancement of biologic activity of the organic drug caused by the presence of the metal ion, possibly due to a longer time of residence of the drug in the organism allowing it to reach the biological targets more efficiently, or due to formation of reactive oxygen species (ROS), among other effects.

b. A decrease in the toxicity of the metal ion towards host cells due to the fact that complexation with organic drugs carries the metal ion to the specific site of action and makes it less readily available for undesired reactions such as inhibition of enzymes, or other damaging reactions leading to a malfunction in the organism.

The work of Williamson and Farrell in 1976 was the first in applying and demonstrate this concept for a tropical disease, trypanosomiasis. [4]

Among other illustrative examples of this approach, it should be mentioned the ferroquine (FQ), in which insertion of a Fe(II) ion in the form of ferrocene into the scaffold of the antimalarial drug chloroquine enhanced the pharmacology of the drug. [5] FQ is being developed by Sanofi-Aventis and entered phase II clinical trials in September 2007. Other example is a ruthenium(II) complex with the antitrypanocidal compound benznidazole, trans-[Ru(Bz) $\left.\left(\mathrm{NH}_{3}\right)_{4} \mathrm{SO}_{2}\right]\left(\mathrm{CF}_{3} \mathrm{SO}_{3}\right)_{2}$, which shows higher hydrosolubility and activity than the free antiparasitic drug. [6] (Figure 2) 
Other advantages of using metal compounds are their pronounced selectivity for selected parasites biomolecules compared to the host biomolecules,[7] and the possibilities they offer to targeted therapies as targeting molecules may be reversibly appended and prodrugs can be developed to deliver highly reactive metal specia in the parasite target while minimising nonspecific interactions.

In the last years, nanotechnology has revolutioned the medicine field by opening novel and promising approaches for drug design, in particular regarding use of nanoparticles $(1-100 \mathrm{~nm})$ as drug delivery vehicles. Despite being liposomes and polymeric particles the most investigated systems to deliver antiparasitic drugs, metal nanoparticles have also emerged as interesting alternative carriers. [8] Furthermore, use of nanoforms of antiparasitic metals like antimony and selenium as alternatives to molecular forms $[9,10]$ has also been recently reported.

In summary, there is a clear need for research in this largely neglected area of medicinal chemistry that is tropical parasitic diseases, and use of metal complexes as possible chemotherapeutic agents arises as a very attractive alternative to tackle this immense problem. However, despite the obvious potential of metal complexes as diagnostic and chemotherapeutic agents, few pharmaceutical or chemical companies have serious in-house research programs that address these important bioinorganic aspects of medicine, which contrast tremendously with the case of purely organic drugs.

The following sections will focus on diverse examples of metal compounds with current or potential applications for leishmaniasis treatment.

\section{Metal compounds as a new generation of leishmanicidal agents. Design strategies}

\subsection{Metal-based drugs for leishmaniasis}

Currently, there is no vaccine against leishmaniasis yet, either purely organic or containing metals. Disease treatment relies solely on chemotherapy. After an intensive revision of the literature, we have found a wide range of metal-containing compounds that are currently used to treat different varieties of leishmaniasis or present a strong antileihsmanial activity and hence potential to be part of a new generation of chemotherapeutic agents with high efficacy and minimal toxicity for the patient. All of them are described in detail in this section.

\subsubsection{Pentavalent antimonials: a (still) unbeatable classic in leishmanicidal therapy}

Antimony-based compounds started to be used a century ago. Trivalent antimonials -Sb(III)were first used, e.g. tartar emetic, which was first reported for treatment of cutaneous leishmaniasis in 1913. But the high toxicity of $\mathrm{Sb}(\mathrm{III})$ compounds and their unstability in tropical climate, led to discovery of pentavalent antimonials - Sb(V)- and in 1920 the $\mathrm{Sb}(\mathrm{V})$ compound urea stibamine emerged as an effective agent against visceral leishmaniasis (kala azar) while being less toxic than the trivalent antimonials. 
<smiles>CCN(CC)CCCC(C)Nc1ccnc2cc(Cl)ccc12</smiles>

Chloroquine (CQ)

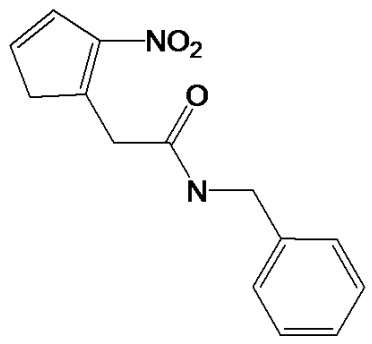

Benznidazole (Bz)<smiles>CC(Nc1ccnc2cc(Cl)ccc12)c1ccccc1CN(C)C</smiles>

Ferroquine (FQ)

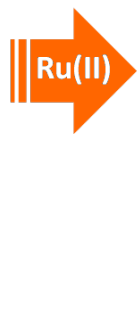

trans- $\left[\mathrm{Ru}(\mathrm{Bz})\left(\mathrm{NH}_{3}\right)_{4} \mathrm{SO}_{2}\right]\left(\mathrm{CF}_{3} \mathrm{SO}_{3}\right)_{2}$

Figure 2. Structures of antimalarial drug chloroquine and antitrypanocidal drug benznidazole, and their respective metallo-derivatives, which show enhanced antiparasitic properties.

Nowadays pentavalent antimonials still constitute the first-line treatment for leishmaniasis. The most commonly used organic salts of $\mathrm{Sb}(\mathrm{V})$ are sodium stibogluconate (Pentostam) and meglumine antimoniate (Glucantime). See figure 3.

However, a significant increase in clinical resistance has been reported for this class of drugs in recent years. In some parts of the world like North East India, the percentage of cases of resistance development is so high (up to 65\%) that these drugs are becoming obsolete.

Although acquired resistance is the most limiting factor for the application of pentavalent antimonials, these drugs present other important drawbacks such as low efficacy for some forms of leishmaniasis and toxic effects (e.g. cardiotoxicity, pancreatitis, anemia and leucopenia). Their toxicity is aggravated by usually required long periods of therapy (up to 4-6 weeks).

Even though antimonials have been in clinical use against leishmaniasis for more than 60 years, their molecular and cellular mechanisms of action are not well understood yet. [11] What is clear is that to be active, antimony has to enter the host cell, cross the phagolysomal membrane and act against the intracellular parasite. By analogy to pentavalent arsenate, it has been 


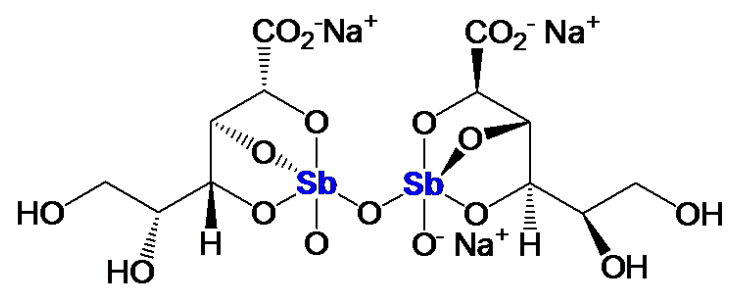

sodium stibogluconate

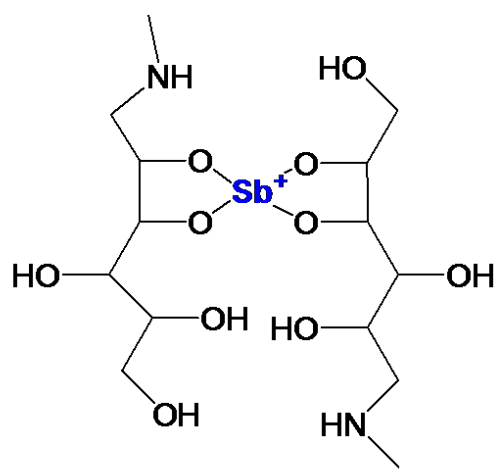

meglumine antimoniate

Figure 3. Chemical structure of sodium stibogluconate (Pentostam) and meglumine antimoniate (Glucantime).

suggested that they enter the cell via a phosphate transporter. Two main models have been proposed to explain the mechanism of action of pentavalent antimonials (Figure 4):

Prodrug model. Recent studies suggest that antimony compromises the thiol redox potential of the cell by inducing efflux of intracellular thiols and by inhibiting trypanothione reductase. Because $\mathrm{Sb}(\mathrm{III})$ is highly active against both stages of the parasite, extra- and intracellular on one hand, and $\mathrm{Sb}(\mathrm{V})$ is active mostly against amastigotes on the other, it is generally accepted that $\mathrm{Sb}(\mathrm{V})$ needs to be reduced to $\mathrm{Sb}(\mathrm{III})$ in order to be active. However, the site and the mechanism of reduction are unclear. Recent results suggest that activation occurs inside macrophages as well as inside parasites (amastigotes). [12] Both reduced glutathione (GSH) and reduced trypanothione $\left(\mathrm{T}(\mathrm{SH})_{2}\right.$ ) have been found to be responsible for non-enzymatic reduction of $\mathrm{Sb}(\mathrm{V})$ to $\mathrm{Sb}(\mathrm{III})$. Other studies have suggested the participation of a parasitespecific enzyme, namely thiol-dependent reductase (TDR1), in the reduction process of $\mathrm{Sb}(\mathrm{V})$ to $\mathrm{Sb}(\mathrm{III})$. Recent crystal structure studies display the mechanism of Leishmania trypanothione reductase (TR) inhibition by $\mathrm{Sb}(\mathrm{III})$. These studies show that trivalent antimony binds the protein active site with high affinity, and strongly inhibits enzyme activity. Metal binds directly to Cys52, Cys57, Thr335 and His461, thereby blocking hydride transfer and trypanothione reduction. Also evidence suggests that the active specia $\mathrm{Sb}(\mathrm{III})$ may interact with zinc-finger proteins by binding Cys residues. The interaction with TR would affect the metabolism of $\mathrm{T}(\mathrm{SH})_{2}$ and induce rapid efflux of intracellular $\mathrm{T}(\mathrm{SH})_{2}$ and $\mathrm{GSH}$ in Leishmania cells. [13] Moreover, the lowering of concentration of intracellular trypanothione in its reduced form $\mathrm{T}(\mathrm{SH})_{2}$, increases the chances for oxidative damage and decreases the disposal of reducing equivalents for DNA synthesis. Sereno et al. found that $\mathrm{Sb}(\mathrm{III})$ induces DNA fragmentation after treating amastigotes of L. infantum at low concentrations of drug, which suggests appearance of late events of apoptosis.[14]

Active $\mathrm{Sb}(\mathrm{V})$ model. According to this model, $\mathrm{Sb}(\mathrm{V})$ would present intrinsic anti-leishmanial activity. It has been shown that sodium stibogluconate, but not $\mathrm{Sb}(\mathrm{III})$, specifically inhibits type I DNA topisomerase by binding the enzyme, thus inhibiting unwinding and cleavage.[15] 
Formation of $\mathrm{Sb}(\mathrm{V})$ complexes with ribonucleosides has been reported, which would be kinetically favored in acidic biological compartments. Moreover, stability constants are consistent with the formation of such a complex in the vertebrate host following treatment with pentavalent antimonial drugs. It is hypothesized that formation of this complex might act as an inhibitor of the Leishmania purine transporters or that, once inside the parasite, this complex interferes with the purine salvage pathway. [16] The formation of these complexes with ribonucleosides might explain as well the depletion of ATP and GTP, as reported previously with sodium stibogluconate. [17]

When antimonials fail, amphotericin B and pentamidine are the recommended second-line treamtent for visceral, cutaneous and mucocutaneous leishmaniasis.However, they are not fully effective either and, additionally,produce toxic side effects. On the other hand, new formulations such as liposomal amphotericin B have been found to be very effective, but its high cost limits its availability to patients.

\section{PRODRUG MODEL}

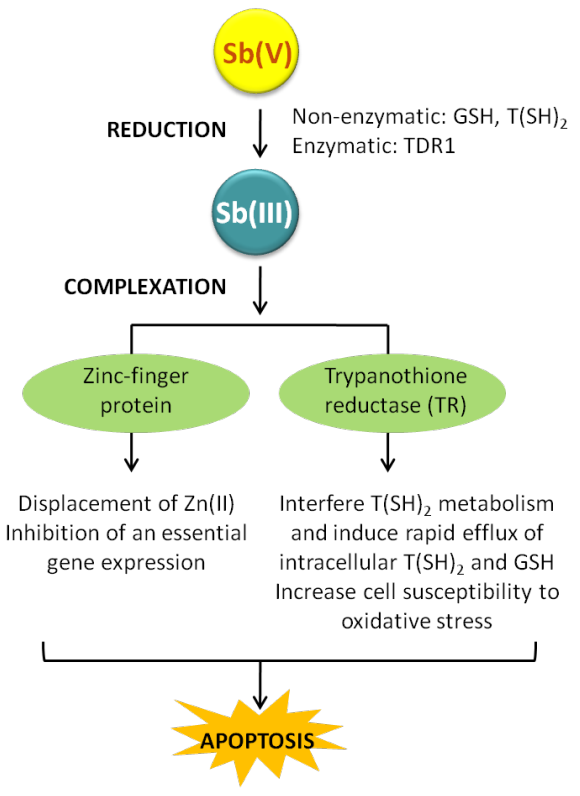

ACTIVE Sb(V) MODEL

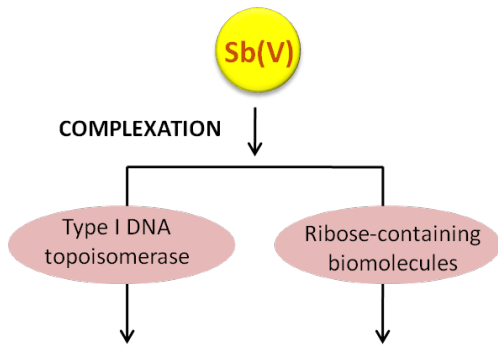

Inhibition of DNA Inhibition of purine transporters unwinding and cleavage Interference with urine salvage pathway Depletion of purine nucleosides

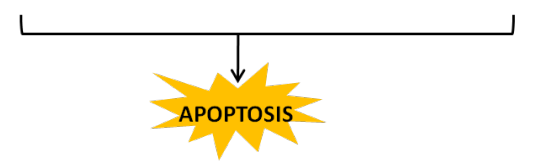

Figure 4. Main models proposed for the mechanism of action of pentavalent antimonials against leishmaniasis.

\subsubsection{Metal complexes of organic drugs: Following the metal-drug synergism approach}

Metal-drug synergism has led to several attempts to develop new potent antiparasitic agents. This approach involves combination of a compound of known antiparasitic activity and a metal in a single molecule. One example is complexation of antileishmanial drug pentamidine with 
$\mathrm{Rh}(\mathrm{I})$ and $\operatorname{Ir}(\mathrm{I})$ to form binuclear complexes of general formula $\left[\mathrm{M}_{2}\left(\mathrm{~L}_{2}\right) \text { (pentamidine) }\right]^{2+}$, where $\mathrm{L}_{2}=1,5$-cyclooctadiene (COD), 1,3,1,5-cyclooctatetraene (COT) or (CO) $)_{2}$. Some of these compounds were found to be more active than the uncomplexed drug pentamidine isethionate. The complex $[\operatorname{Ir}(\mathrm{COD})$ (pentamidine) $]\left[\mathrm{BPh}_{4}\right]$ exhibits the same in vitro activity as free pentamidine, but its in vivo activity reaches $23 \%$ and $32 \%$ of parasite suppression for L. donovani and L. major, respectively, under conditions where pentamidine isethionate is inactive. The related compound $\left[\mathrm{Ir}_{2}(\mathrm{COT})_{2} \text { (pentamidine)][alizarin red }\right]_{2}$ showed to be at least twice as active as pentamidine isethionate against amastigotes of $L$. donovani and synergistic effect was observed when this complex was administered in combination with pentamidine, amphotericin B or paromomycin. [18]

Other metal-drug synergy-based strategies make use of diverse chemotherapeutic targets such as sterol 14-demethylases by attaching azole-type sterol biosynthesis inhibitors (SBIs) such as clotrimazole (CTZ) and ketoconazole (KTZ), to a metal-containing fragment. For example, compound $\left[\mathrm{Ru}\left(\eta^{6}\right.\right.$-p-cymene $\left.) \mathrm{Cl}_{2}(\mathrm{CTZ})\right]$ shows an enhancement of the activity of $\mathrm{CTZ}$ by a factor of 110 against L. major promastigotes, resulting in low nanomolar lethal doses. In addition, this $\mathrm{Ru}(\mathrm{II})$ compound does not exhibit any appreciable toxicity toward human osteoblasts when assayed up to $7.5 \mu \mathrm{M}$, which translates into excellent selectivity indexes higher than 500. This compound also significantly inhibited the proliferation of intracellular amastigotes of L. major in infected intraperitoneal mouse macrophages $\left(\mathrm{IC}_{70}=29 \mathrm{nM}\right)$. In vivo testing and detailed mechanistic studies of these ruthenium-CTZ complexes are currently in progress.[19] Likewise, a series of $\mathrm{Ru}(\mathrm{II})$ complexes with $\mathrm{KTZ}$ have recently been synthesized: cis,fac-[RuCl$\left.{ }_{2}(\mathrm{DMSO})_{3}(\mathrm{KTZ})\right], \quad$ cis-[RuCl$\left.{ }_{2}(\mathrm{bipy})(\mathrm{DMSO})(\mathrm{KTZ})\right], \quad\left[\mathrm{Ru}\left(\eta^{6}\right.\right.$-p-cymene) $\left.\mathrm{Cl}_{2}(\mathrm{KTZ})\right]$,

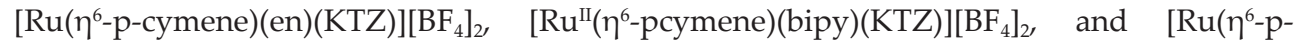
cymene)(acac)(KTZ)][BF $\left.{ }_{4}\right]$. They showed a marked increase of the activity against promastigotes and intracellular amastigotes of L. major when compared with free KTZ or with similar ruthenium compounds not containing KTZ. Interestingly, selectivity of some of these compounds toward Leishmania parasites in relation to normal human cells was also higher than selectivities of the individual constituents of the drug. Hydrolysis of the chloride ligands to form cationic aqua species appears to be a prerequisite for biological activity, and dissociation of KTZ probably occurs but only on further interactions of the active species with biomolecules within the parasite cell. Authors relate the antiparasitic activity to a combination of the SBI action of dissociated KTZ and the ability of the nitrogen-containing ligands on the remaining ruthenium fragment to promote interactions with DNA through hydrogen bonding or by $\pi-$ stacking interactions. [20]

Other metal ions like Pt(II), Rh(I) or Os(III) have been used to obtain organometallic compounds with ligands derived from benzothiazole, a compound of which some derivatives have shown promising antimicrobial, antifungus and antiparasite activity. The obtained compounds were active against promastigotes and amastigotes of $L$. donovani by targeting different biochemical pathways: [cis-[Pt(da)(2,5-dihydroxybenzenesulfonate) $\left.{ }_{2}\right](\mathrm{da}=1,2$-diaminocyclohexane), $\left[\mathrm{Ru}(\mathrm{CO})_{2}(2\right.$-aminobenzothiazole $\left.)\right],\left[\mathrm{Ru}(\mathrm{CO})_{2}(2\right.$-methylbenzothiazole $\left.)\right],[21]$ and a series of dithiocarbamate complexes with formula $[\mathrm{Os}(\mathrm{L})]$ where $\mathrm{L}=$ nitroimidazole, dinitroimidazole, benznidazole. Osmium complexes clearly inhibited DNA, RNA and protein 
synthesis, as well as enzymatic activities of succinate dehydrogenase, malate dehydrogenase and pyruvate kinase. [22]

Apart from vanadium compounds ability to exert different insulin-mimetic and antidiabetic effects, it has been recently proved that vanadium also offers interesting chemical and biochemical properties for the development of antiparasitic drugs. Noleto et al. combined the oxovanadium(IV) core with the antileishmanial compound galactomannan (GMPOLY), isolated from southern Brazil lichen Ramalina celastri. [23] Complexation highly increased leishmanicidal effect of galactomannan on amastigotes of L. amazonensis infecting peritoneal macrophages. This effect of GMPOLY on amastigotes could be attributed to the activation of the nitric oxide pathway. Nitric oxide is secreted by macrophages in response to IFN- $\gamma$ (interferon $\gamma$ ) stimulation and it is regulated by tyrosine phosphatase events. Since the effect detected for GMPOLY oxovanadium(IV) complex occurred at concentrations where GMPOLY was non active, authors suggested the involvement of oxovanadium(IV) ion in the anti-parasite action.

\subsubsection{Targeting cysteine proteases}

Cysteine proteases have been found to play multiple roles in parasitic life cycles including nutrition, host invasion, protein processing, and evasion of the host immune response. In fact, there is an abundance of data to suggest that parasite cysteine proteases represent valid drug targets. For example, it was shown that an inhibitor of cathepsin Blike cysteine protease of L. major, $\mathrm{cpB}$, inhibited parasite growth in vitro and ameliorated the pathology associated with a mouse model of leishmaniasis. [24] Since cysteine proteases found in Leishmania and T. cruzi have similarities to mammalian cathepsins B and L, the latter ones have been used as models to study the bioactivity of diverse metallic compounds. Cyclometallated gold, palladium, and rhenium derivatives have displayed cathepsin B inhibitory ability against cathepsin B and also similar order activity against the corresponding parasite enzyme cpB. These compounds have also shown growth inhibition of extracellular promastigotes of L. major, L. mexicana and L. donovani (see figure 5 and table 1). [25]

\subsubsection{Vanadium compounds and their interaction with protein tyrosine phosphatases}

Peroxovanadium compounds have shown potent inhibitors of protein tyrosine phosphatases and inducers of antileishmania effects like ROS and NO. Treatment of infected mice with bisperoxovanadium-1,10-phenanthroline or bis-peroxovanadiumpicolinate completely controlled progression of leishmaniasis in a NO-dependent manner. After injection, compounds rapidly triggered expression of inducible NO synthase in liver of mice infected with L. major. In vivo functional and immunological events associated with this peroxovanadium protective process have been identified. More recently, three dinuclear triperoxovanadate complexes, two mononuclear diperoxovanadate complexes with aminoacids or dipeptides as ancillary ligands and bis-peroxovanadate have been tested for their ability to kill Leishmania parasites in vitro, being $\mathrm{K}\left[\mathrm{VO}\left(\mathrm{O}_{2}\right)_{2}\left(\mathrm{H}_{2} \mathrm{O}\right)\right]$ the most potent one. Combined administration of the latter with sub-optimal doses of sodium antimony gluconate on BALB/c mice experimentally 
A<smiles>CC(=O)O[Al]1(OC(C)=O)c2ccccc2Cc2cccc[n+]21</smiles>

1

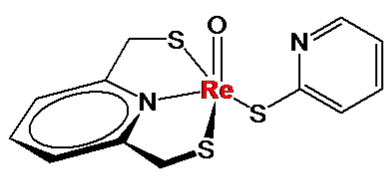

4<smiles>CCCCO[P@]1(C(C)=O)SC2c3cccc(c31)C[SH]2CCCC</smiles><smiles>O=S1SCCS(Cl)(Cl)S1</smiles>

5<smiles>COc1ccc(S(=O)(=O)N2CCSCC2CC2CCCCC2)cc1</smiles>

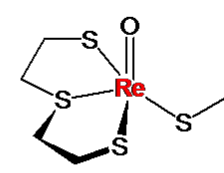

6

$\mathbf{B}$
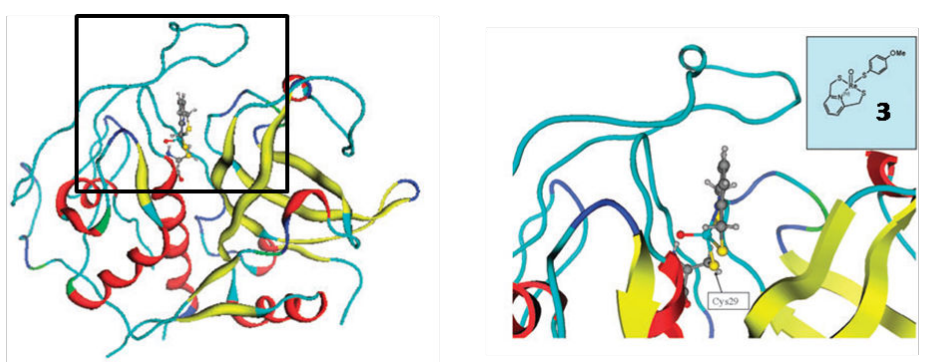

Figure 5. A) Structures of metal complexes with antileishmanial activity via inhibition of parasite cysteine proteases: (1) diaceto [2-[(2-pyridinyl-kN)methyl] phenyl-kC] gold(I), (2) aceto [2,6-bis [(butylthio-kS)methyl]-phenyl-kC] palladium(II), (3) (p-methoxyphenylthiolato-S) [2,6-bis[(mercapto-kS)methyl] pyridine-k $\left.N^{1}\right]$ oxorhenium $(\mathrm{V}),(4)(2(1 H)$-pyridinethionato-k $\left.S^{2}\right)\left[2,6\right.$-bis [(mercapto-kS)-methyl] pyridine- $\left.\mathrm{k} N^{1}\right]$ oxorhenium $(\mathrm{V}),(5)$ chloro [2,2'-(thio-kS)bis [ethanethiolato- $\mathrm{k} S$ )] ] oxorhenium(V), (6) (methanethiolato) [2,2'-(thio-kS)bis [ethanethiolato-kS)]] oxorhenium(V). B) Hypothetical model of oxorhenium( $(\mathrm{V})$ complex 3 binding to the active site cysteine of cathepsin B. Adapted from Ref. [25].

\begin{tabular}{lcccccc}
\hline Compound & $\mathbf{1}$ & $\mathbf{2}$ & $\mathbf{3}$ & $\mathbf{4}$ & $\mathbf{5}$ & $\mathbf{6}$ \\
\hline Cat B & 1.29 & 0.40 & 6.51 & 0.12 & 0.0088 & 1.26 \\
\hline L. Major cpB & 1.7 & 2.1 & 1.0 & 0.07 & 0.2 & $" />10$ \\
\hline
\end{tabular}

Table 1. Inhibitory effect of metal compounds 1-6 against mammalian cathepsin B and cathepsin B-like cystein protease of L. major. Results expressed as $I C_{50}(\mu \mathrm{M})$. [25]

infected with antimony resistant $L$. donovani was highly effective in reducing the organ parasite burden. The effect was mainly associated with generation of ROS and nitrogen species that could kill intracellular parasites. 


\subsubsection{DNA-metallointercalators are not only to fight cancer}

As the metabolic pathways of kinetoplastid parasites are similar to those of tumor cells, it has been proposed that compounds which efficiently interact with DNA in an intercalative mode could also show anti-trypanosomatid activity. [26] Based on this hypothesis, some work has been carried out on design of metallointercalators as anti-leishmania drugs, including metals of pharmacological interest. It has been found that certain DNA intercalating drugs which have potent trypanocidal action, such as ethidium, acriflavine, and ellipticines, inhibit the DNA topoisomerases. These enzymes thus may represent another potential target for DNAintercalating trypanocidal metallodrugs.

DNA-intercalating metal complexes with potential leishmanicidal activity are generally made up of metals of known clinical application such as platinum, copper, silver and gold with planar polyaromatic ligands such as dppz (dipyrido[3,2-a:2',3'-c]phenazine) and dpq (dipyrido[3,2-a:2',3'-h]quinoxoline). Figure 6. Copper complexes with dppz and dpq ligands, $\left[\mathrm{Cu}(\mathrm{L})_{\mathrm{n}}\left(\mathrm{NO}_{3}\right)_{2-\mathrm{n}}\right]\left(\mathrm{NO}_{3}\right)_{\mathrm{n}}$ where $\mathrm{L}=\mathrm{dppz}$ or dpq (Fig. 6) have shown activity against Leishmania braziliensis (causative of the muco-cutaneous mode of the disease), and it has been demonstrated that their action is related to their ability to interact with DNA. $\left[\mathrm{Cu}(\mathrm{dppz})_{2}\right]\left(\mathrm{NO}_{3}\right)_{2}$ was the most effective complex in this series, and the activity order was $\left[\mathrm{Cu}(\mathrm{dppz})_{2}\right]$ $\left(\mathrm{NO}_{3}\right)_{2}>\left[\mathrm{Cu}(\mathrm{dppz})\left(\mathrm{NO}_{3}\right)\right]\left(\mathrm{NO}_{3}\right)>\left[\mathrm{Cu}(\mathrm{dpq})_{2}\right]\left(\mathrm{NO}_{3}\right)_{2}>\left[\mathrm{Cu}(\mathrm{dpq})\left(\mathrm{NO}_{3}\right)\right]\left(\mathrm{NO}_{3}\right)$. [27]

Among the most effective complexes is $\left[\mathrm{Au}(\mathrm{dppz})_{2}\right] \mathrm{Cl}_{3}$. This complex induced a dose dependent antiproliferative effect with a minimal inhibitory concentration (MIC) of $3.4 \mathrm{nM}$ and lethal doses $\mathrm{LD}_{26}$ of $17 \mathrm{nM}$ at $48 \mathrm{~h}$. This strong in vitro activity against L. mexicana could be related to their ability to interact with DNA through an intercalative mode. Also, preliminary ultrastructural studies using transmission electron microscopy carried out with treated parasites at a sublethal concentration ( $\mathrm{IC}_{7}=0.34 \mathrm{nM}$ for $24 \mathrm{~h}$ ) showed polynucleated cells with DNA fragmentation and drastic disorganization of the mitochondria. [28]

Several years ago, a DNA metallointercalator $\left(2,2^{\prime}: 6^{\prime} 2^{\prime \prime}\right.$-terpyridine) platinum showed a remarkable antileishmanial activity, causing complete growth inhibition of Leishmania donovani amastigotes at $1 \mu \mathrm{M}$ concentration.[29] This complex exploits simultaneous DNA intercalation of terpyridine and platinum(II) binding to the enzyme active site. The highest activity against $L$. donovani was found for the case of $p$-bromophenyl substituents in 4 'terpyridine position, and $\mathrm{NH}_{3}$ as the ancillary hydrolysable ligand.[18]

Various DNA-intercalating organic ligands, have also been bound to vanadium ions. Although the potentiality of vanadium compounds in medicinal chemistry and medicinal applications has been extensively explored, work on vanadium compounds for treatment of some parasitic diseases of high incidence in human health has only arisen in a systematic way in recent years. [30] Benítez et al. obtained a series of oxovanadium complexes combining the aromatic planar polycyclic system 1,10-phenanthroline (phen) and tridentate salicylaldehyde semicarbazone derivatives as ligands, $\left[\mathrm{VO}\left(\mathrm{L}^{1}-2 \mathrm{H}\right)(\right.$ phen $\left.)\right]$ and $\left[\mathrm{VO}\left(\mathrm{L}^{2}-2 \mathrm{H}\right)(\right.$ phen $\left.)\right]$, where $\mathrm{L}^{1}=2$-hydroxybenzaldehyde semicarbazone and $\mathrm{L}^{2}=2$-hydroxy-3-methoxybenzaldehyde semicarbazone. These compounds were active against Leishmania parasites showing low toxicity on mammalian cells. In addition, they showed cytotoxicicity on human promyelocytic leukemia HL-60 cells with 


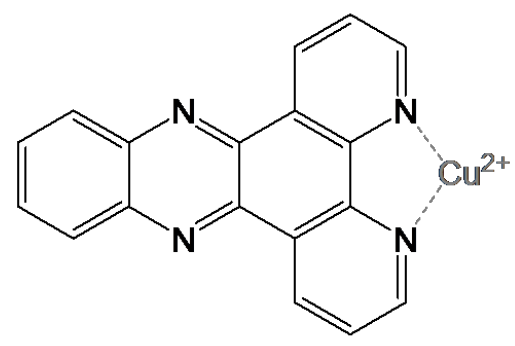

dppz

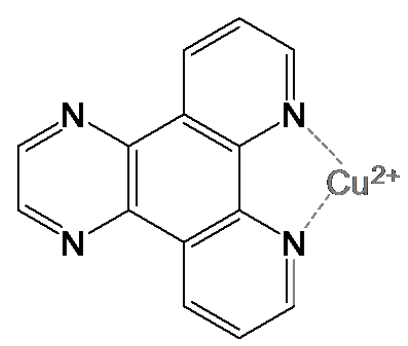

$d p q$

Figure 6. Structures of dppz (dipyrido[3,2-a:2',3'-c]phenazine) and dpq (dipyrido[3,2-a:2', 3'-h]quinoxoline).

$\mathrm{IC}_{50}$ values of the same order of magnitude as cisplatin. Their interaction with DNA was demonstrated and studied by different techniques, suggesting that this biomolecule could be one of the potential targets for activity either in parasites or in tumor cells. [31]

\subsubsection{Zinc sulphate against cutaneous leishmaniasis: The privilege of simplicity}

Since zinc sulphate administered orally has been used in the last decades in medicine and dermatology, [32] then its use as an oral therapy for cutaneous leishmaniasis has appeared recently as an important addition to the armamentarium of antileishmanial drugs.

In vitro sensitivities of L. major and L. tropica strains to zinc were reported to be higher than those to pentavalent antimony, and these data were confirmed on mice. Zinc sulphate was also delivered intralesionally with success in cutaneous leishmaniasis. It is been suggested that oral zinc might not only affect directly to the parasite but also to macrophages function. Also it could have immunomodulatory effect (including T-lymphocytes), and help wound-healing. [33]

More recently, zinc sulphate was orally administered to Iraqi patients suffering from parasitologically confirmed cutaneous leishmaniasis. The species was not identified but it is known that only L. major and L. tropica are present in Iraq. This salt showed very promising cure rates (96.9\%) against cutaneous leishmanaisis in a 45-days treatment with oral daily doses of $10 \mathrm{mg} /$ $\mathrm{kg}$. After a comparative study between oral zinc sulfate and meglumine antimoniate in the treatment of cutaneous leishmaniasis, it was suggested that systemic antimonial injections in cutaneous leishmaniasis treatment were better than zinc sulphate but oral administration of zinc sulphate makes it cheaper, more convenient its consumption, and nearly close cure percentage to systemic meglumine antimoniate injections without serious side effects. However at the moment zinc sulphate therapeutic effects should be confirmed by a greater sample volume. [34] Nevertheless, reported studies suggest that antileishmanial effect of zinc may result, partially or entirely, from inhibition of enzymes that are necessary for the parasites' carbohydrate metabolism and virulence. [35] 


\subsubsection{Selenium and the key role of antioxidants in disease}

Selenium is an important and potent antioxidant in cells. Selenium compounds like selenites and selenates have strong inhibitory effects particularly on mammalian tumor cell growth. What is more, the nutritional deficiency of this essential trace metal may inhibit initiation and post-initiation phases of chemically induced mammary carcinogenesis and expression of some viruses, and it is important for optimal functioning of the immune system. [36]

Compounds of this metal have been reported to control human malaria if used in combination with vitamin E. [37] In vitro studies have shown that sodium selenite can inhibit Leishmania donovani growth although the mechanism of action is not clear yet. [38] Some authors have suggested that selenium has an important role in the pathophysiologic processes of cutaneous leishmaniasis, and that decreasing levels of this metal may be a host defense strategy of the organism against cutaneous leishmaniasis infection. Lack of selenium leads to a decrease of GSH-Px enzyme activity (it degrades $\mathrm{H}_{2} \mathrm{O}_{2}$ ), leading to increased amounts of hydroperoxides to kill protozoa as a host defense strategy.

\subsubsection{Triazolopyrimidines and their metal complexes: Mimicking the nature}

Triazolopyrimidines are purine analogues that have attracted much pharmaceutical interest during last decades. The most widely known derivative is the simple molecule Trapidil or Rocornal, a clinically used antiischemic and cardiatonic agent which acts as a platelet-derived growth factor (PDGF) antagonist and as a phosphodiesterase inhibitor. [39] This family of compounds have also found interesting applications as antipyretic, analgesic and antiinflammatory, herbicidal, fungicidal agents with about 200 relevant patents. For example, 2arenesulfonamido triazolopyrimidines were tested as leishmanicides showing some of them similar in vitro activity than pentamidine against $L$. donovani (Figure 7 ).

a)

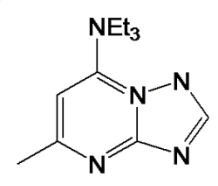

b)

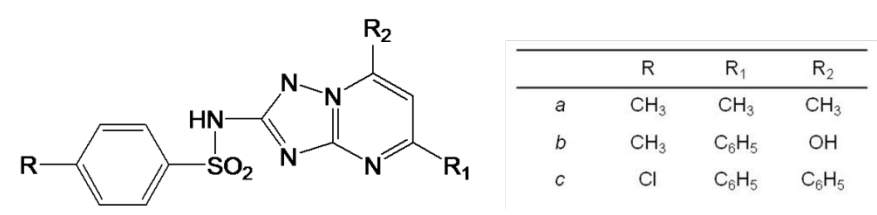

Figure 7. Structures of triazolopyrimidine drugs: a) the anticoagulant drug Trapidil; b) a series of leishmanicidal derivatives.[39]

The biological activity of this family of organic compounds has led to investigating their coordination chemistry with the aim to develop new drugs with enhanced leishmanicidal activity and selectivity towards the parasites. Recently our group developed a series of transition metal complexes containing 1,2,4-triazolo[1,5-a]pyrimidines with high antiproliferative activity and extremely high selectivity indexes (see section 3). Studies revealed that apart from being all of them active in vitro against both extracellular and intracelular forms of $L$. infantum and L. braziliensis, these compounds are not toxic towards the host cells and are effective at lower concentrations than the drug used as a reference, Glucantime.[40] In the 
following section, we will present a case study in which our latest findings of our research with triazolopyrimidine metal complexes are described.

\subsubsection{Nanoparticles: a promise for the future}

A vast array of intriguing nanoscale particulate systems capable of targeting different cells and extracellular elements in the body to deliver drugs, genetic materials and diagnostic agents have been developed in the last years. Currently, antiparasitic delivery via nanosized particles is at the forefront of the research in this area. Liposomes and polymeric nanoparticles are the best studied nanosystems for evaluating antileishmania activity of compounds like amphotericin B or pentamidine. [41]

But nanosized metal particles are also emerging as promising antiparasitic agents. In recent studies it was determined that metal nanoparticles possess effective antimicrobial activities due to their unique properties and large surface areas. Moreover, metal nanoparticles are capable of producing reactive oxygen species (ROS), which would be able to kill parasites and other infectious agents.

Use metal of metal nanoparticles against Leishmania has followed two main approaches:

a. As antiparasitic drug carriers. Nano-bioconjugate gold has recently been conceived as a stratagem against macrophage-infested leishmanial infections. One example is the functionalization of gold nanoparticles with the flavonoid quercetin, reported as one of the most powerful leishmanicidal among all plan flavonids tested so far. [8] This flavonoid inhibits synthesis of parasite DNA by inhibition of topoisomerase II mediated linearization of kDNA. Quercetin in addition can chelate iron and then limit availability of this metal for ribonucleotide reductase during DNA synthesis. On the other hand, gold nanoparticles as such can cause impairments in parasite oxygen metabolism.

Quercetin functionalized gold nanoparticles showed to be effective against L. donovani promastigotes and amastigotes. They were also effective against drug resistant strains with a very high selectivity index. A synergistic effect was considered by the authors as a possible reason for the higher activity of the nanoconjugate related to the free quercetin.

b. As antiparasitic administration nanoforms. Because of the larger surface area of nanoparticles, they are more reactive and thus chemotherapeutic properties of a metal with antiparasitic activiy would be enhanced for its nanoform.

Selenium, for example, is a bioactive metal as it has antioxidant, cancer preventing, and antiviral activities. [37] Beheshti et al. prepared biogenic selenium nanoparticles, in this case, biosynthesized by Bacillus sp. MSh-1 and tested their in vitro and in vivo activity against Leishmania major. The particles showed antiproliferative activity against promastigote and amastigote forms of L. major and limited localized cutaneous leishmaniasis in animal model. These results present this kind of particles as novel therapeutic agents for treatment of the localized lesions typical of cutaneous leishmaniasis. However further studies are needed to investigate the mechanism of action of these Se NPs.[9] 
Antimony sulfide NPs $\left(\mathrm{Sb}_{2} \mathrm{~S}_{5}\right)$, obtained also by green synthetic methods, proved to be effective on proliferation of promastigote forms of L. infantum and can induce apoptosis in promastigotes. [10]

The capability of metal nanoparticles to generate ROS and their potential use as leishmanicidal agents have also been explored. This is the case of silver nanoparticles, which have shown to be able to produce high amounts of ROS independently of the host cells. In vitro effects of AgNPs against promastigotes and amastigotes of Leishmania tropica were investigated. In order to increase the amount of ROS that are generated, AgNPs were irradiated with UV light which enhanced their antileishmanial effects without affecting host cells. [42]

\subsection{Strategies for the design of new metal-based leishmanicidal drugs}

To address the need for new, cost-effective metal-based leads for chemotherapy of leishmaniasis, different strategies of structure-based drug design have been applied so far. Four main strategies may be identified along revision in section 2.1:

\subsubsection{Antitumoral activity implies antiparasitic activity}

This strategy is based on the knowledge that highly-proliferative cells such as kinetoplastid parasites and tumor cells show metabolic similarities that lead in many cases to a correlation between antitrypanosomal and antitumor activities.[4] In this sense, use of metal complexes which have previously shown antitumoral activity, or synthesis of new metal complexes with ligands bearing activity could be a promising approach towards development of new agents against protozoa like Leishmania. A good correlation between antitumor and trypanostatic properties of several metal-based drugs has already been observed.

\subsubsection{Metal-drug synergism approach}

Perhaps one of the most popular strategies to develop new antiparasitic drugs consists on using an established antiparasitic drug as scaffold for the inclusion of a metal centre, either via direct coordination to the drug or by binding a metal complex. This way an enhancement of the drug pharmacological properties is pursued and resistance mechanism might be circumvented. See section 2.1.2.

\subsubsection{Delivery nanovehicles}

In finding innovative parasite-specific formulations, established but deficient drugs might be optimised by using drug delivery systems, in order to enhance their efficiency and reduce negative side effects at relatively low cost. Antiparasitic efficacy of drugs already in clinical use might be significantly improved by the adaptation of a new drug formulation. Use of nanocarriers to deliver established metal-based drugs such as antimonials would be both costeffective and the quickest way to produce effective results. New drug formulations like liposomes for other drugs like amphotericin B (Ambisome) have been successfully developed for treating visceral leishmaniasis. 
On the other hand, use of metal-based nanosystems as drug carriers, e.g. noble metal nanoparticles, might provide additional advantages such as the possibility for diagnosis by imaging techniques and the combined effect of producing ROS as it is the case for silver. ROS can induce oxidative stress, DNA damage, alkylation of target proteins and eventually apoptosis of the parasite.

In order to inhibit Leishmania parasites with a ROS-based treatment, these oxygen derivatives must be produced in a physical way rather than in an enzymatic way that can be blocked by parasites. Metal nanoparticles are able to produce high amounts of ROS, as they are more reactive than the corresponding bulk metal (see example of AgNPs in section 2.1.9).

Nanocarriers also offer the possibility to specifically target the parasites by attaching appropiate targeting molecules onto their surface. This way side effects to the host would be minimised. In addition, drug delivery vehicles such as nanoparticles allow prompt interactions with biomolecules present within as well as on the surface of the cell and may be tuned into different sizes to get the optimal uptake rate and blood circulation times of the drug.

\subsubsection{Specifically targeted drugs: Metal inhibitors of parasite enzymes and DNA-binders}

Recent advancements in molecular biology have identified a few parasite targets that are likely to be very sensitive to metal-based compounds. These targets usually are enzymes, some of them bearing free thiols at their active sites that manifest a high propensity to react with soft Lewis acids, i.e. metal ions such as $\mathrm{Ag}(\mathrm{I}), \mathrm{Au}(\mathrm{III})$ or $\mathrm{Zn}(\mathrm{II})$. Therefore these parasite targets will be susceptible to strong and selective inhibition by this kind of metals. This is the case of dithiol reductases like trypanothione reductase $\left(\mathrm{T}(\mathrm{SH})_{2}\right)$, which have been shown to play a key role in the Leishmania metabolism (see Section 2.1.1) and therefore constitute primary targets for metal compounds. Cysteine proteases, such as cathpesin L-like or cathepsin B-like, are another example of proteins with thiol-containing active sites and thus responsive to inhibition by metal compounds. Inhibitors that would effectively target both types of cysteine proteases in Leishmania, while maintaining some selectivity versus homologous host enzymes, would be ideal drug leads.

Regarding DNA interaction, previous studies have shown that DNA-binding metal compounds such as cisplatin display antiparasitic activity. These findings along with the observation that many antiparasitic drugs bind to DNA, have led to propose that in general every DNA interacting compound is potentially active against parasites.

Therefore DNA-intercalating molecules have been used as ligands to form metal complexes showing antiparasitic activity. Intercalating ligands are usually polyaromatic systems with two or more donor atoms in close disposition to "chelate" metal ions. These ligands would not only be responsible for interaction of the metal compound with DNA but also they could act as carriers of the metal, increasing interaction of complexes with DNA by minimizing exposure of metal to inactivating cellular nucleophiles such as thiols. 


\section{Case study: Evaluation of the chemotherapeutic potential of metal complexes containing nucleobase-analogues against Leishmania infantum and Leishmania braziliensis}

In this section we will describe some of our latest findings, which have been published recently. [40] Through this case study, we seek to provide the reader with an useful insight on our research, which is aimed at the rational design of new biomimetic metal-based systems as potential antiparasitic agents. Our research activity can be summarized in the following tasks:

a. Study of the interaction of a series of purine analogs, namely 1,2,4-triazolopyrimidines, with a wide range of metal ions, mainly from the first and second transition series.

b. Based on the coordination properties of triazolopyrimidine derivatives, design and synthesize new metal complexes showing structural and physical properties such as photoluminescent or magnetic properties that might be of interest for further applications.

c. Evaluate their in vitro activity against Trypanosoma cruzi and different species of Leishmania spp. Studies with T. cruzi are complemented with in vivo assays (murine model) for the most active compounds.

d. Analyze possible structure-activity correlations and investigate mechanism of action.

\subsection{Transition metal complexes with 1,2,4-triazolo[1,5-a]pyrimidines}

1,2,4-triazolopyrimidines are bicyclic heterocycles that are formed from the condensation of a ring of 1,2,4-triazole and another one of pyrimidine. Depending on the relative orientation of both rings, four different isomeric families can arise: 1,2,4-triazolo[1,5-a]pyrimidines, 1,2,4triazolo[1,5-c]pyrimidines, 1,2,4-triazolo[4,3-a]pyrimidines, and 1,2,4-triazolo[4,3-c]pyrimidines. Among them, 1,2,4-triazolo[1,5-a]pyrimidine derivatives are the most stable thermodynamically and, because of this, the object of our present studies.

In previous works, 1,2,4-triazolo[1,5-a]pyrimidines have proved to be excellent ligands for a wide range of transition metal ions. [43] This fact is due to their, at least, three coordination positions, N1, N3 and N4, which can lead to several coordination modes. The coordination capability of these derivatives can be increased by the presence of heteroatoms as ringsubstituents. However, a systematic revision on the existing results indicates a clear trend of these ligands to coordinate monodentately by N3, followed by N3,N4-bidentate and N1,N3bidentate bridging modes (Figure 8).

The rich coordination chemistry of these derivatives has led in the last years to a great variety of multidimensional coordination compounds showing interesting properties, especially from the magnetic and biological viewpoints.

In addition, the biomimetic character of 1,2,4-triazolo[1,5-a]pyrimidines with purine nucleobases confers a potential biological activity to these derivatives and to their metal complexes, which can be used for therapeutic aims. Our studies have revealed the high potential of this kind of compounds for acting as leishmanicidal agents. 
a)

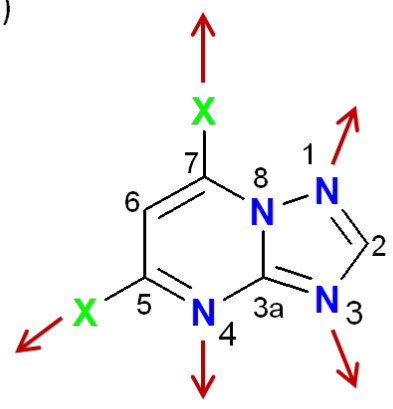

1,2,4-triazolo[1,5-a]pyrimidines b)<smiles>c1ncc2nc[nH]c2n1</smiles>

purines

Figure 8. Basic structure of 5,7-substituted 1,2,4-triazolo[1,5-a]pyrimidines (a) and purines (b). Numbering scheme and possible binding sites to metal ions are also depicted for triazolopyrimidines. $\mathrm{X}=$ donor atom (N, O, S, etc.)

Herein we report the results obtained with three of the most promising metal compounds we have obtained so far: $\left[\mathrm{Cu}(\mathrm{HmtpO})_{2}\left(\mathrm{H}_{2} \mathrm{O}\right)_{3}\right]\left(\mathrm{ClO}_{4}\right)_{2} \mathrm{H}_{2} \mathrm{O}(1),\left\{\left[\mathrm{Cu}(\mathrm{HmtpO})_{2}\left(\mathrm{H}_{2} \mathrm{O}\right)_{2}\right]\left(\mathrm{ClO}_{4}\right)_{2}\right.$ $2 \mathrm{HmtpO}\}_{\mathrm{n}}(2)$ and $\left.\left\{\mathrm{Co}(\mathrm{HmtpO})\left(\mathrm{H}_{2} \mathrm{O}\right)_{3}\right]\left(\mathrm{ClO}_{4}\right)_{2} 2 \mathrm{H}_{2} \mathrm{O}\right\}_{\mathrm{n}}$ (3), Figure 9. All of them contain the neutral form of 5-methyl-1,2,4-triazolo[1,5-a]pyrimidin-7(4H)-one (HmtpO) and perchlorate as counteranion. The three compounds show different topology and dimensionality. Compound 1 is a monomeric complex in which HmtpO shows both N3 monodentate and N1,O71 bidentate modes; compound 2 is a two-dimensional framework in which HmtpO ligand shows an N3,O71 bidentate bridging mode; and the structure of compound 3 consists of onedimensional chains in which HmtpO displays an N1,N3,O71 tridentate bridging mode. The structural diversity of these compounds is mainly due to the mode of the triazolopyrimidine ligand.

As depicted in Figure 9, the compounds 1-3 were synthesized by mixing their corresponding metal perchlorate salts with $\mathrm{HmtpO}$ derivative in aqueous media and bringing to reflux for 30 min before acidification with $\mathrm{HCl}$. In all cases, compounds were isolated as crystals from their respective solution after several days standing at room temperature. Obtention of single crystals allowed to determine their crystal structure by $\mathrm{X}$-ray analysis and their characterization was completed by elemental and thermal analysis (thermogravimetry and differential scanning calorimetry), and spectroscopic techniques such as FTIR and UV-Vis. Magnetic studies indicate that compound 1 exhibits simple paramagnetism in 2-300 K while the overall behaviour of 2 and 3 corresponds to weak ferromagnetically and antiferromagnetically coupled systems, respectively. [44]

\subsection{In vitro antiproliferative activity against promastigote forms (extracellular forms) and toxicity against a mammalian host cell model}

Firstly we evaluated the toxic activity of the free triazolopyrimidine compound HmtpO and its $\mathrm{Cu}(\mathrm{II})$ and $\mathrm{Co}(\mathrm{II})$ complexes 1-3 against promastigotes of two species of Leishmania (L. 


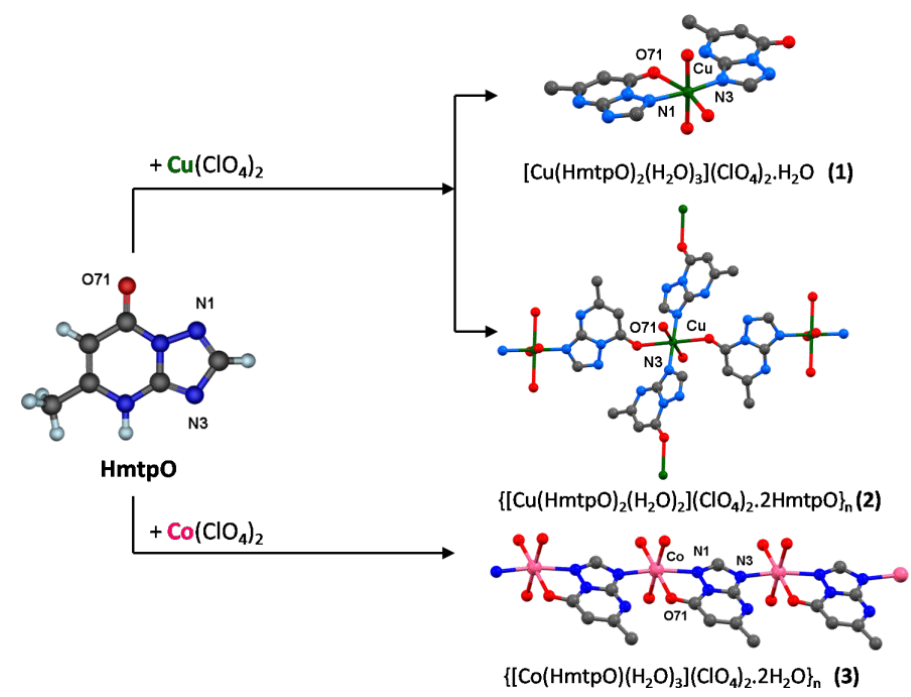

Figure 9. Synthetic scheme and structures of triazolopyrimidine derivative HmtpO and its metal complexes 1-3. Please note that the graphs of 1-3 correspond only to the cationic part of the metal compounds.

infantum and L. braziliensis). $\mathrm{IC}_{50}$ values registered after $72 \mathrm{~h}$ of exposure are shown in Table 2, including Glucantime as reference drug. Antileishmanial activity of metal complexes 1-3, expressed as $\mathrm{IC}_{50}$, was similar to that found for Glucantime for both L. infantum and $L$. braziliensis. In contrast, the free derivative HmtpO is significantly less active than its metal compounds.

To evaluate toxicity on the host, J774.2 macrophages (mammalian cells) were used as cell model. Cytotoxic studies showed that metal complexes 1-3 are much less toxic than Glucantime and the free HmtpO derivative (Table 2).

On the other hand, selectivity and thus efficacy of assayed compounds towards parasite cells was evaluated and quantified by using the selectivity index (SI). This parameter is defined as the cocient between $\mathrm{IC}_{50}$ for cells and $\mathrm{IC}_{50}$ for parasites. A value greater than 1 is considered more selective for activity against parasites, and a value less than 1 is considered more selective for activity against cells.[45] SI of these derivatives was 30-fold or more higher than SI of Glucantime and HmtpO. These results are indicative of the higher potential of metal compounds 1-3 as antiparasitic agents compared with the current treatments, in this case Glucantime. Moreover it is evident that the presence of the metal ion in the scaffold enhances significantly triazolopyrimidine derivative activity and selectivity. This example constitutes another proof of the validity of the metal-drug synergism approach.

\subsection{Effects on the infection rate and the intracellular replication of the amastigote forms}

Most studies on in vitro biological activity of new compounds against Leishmania spp. are performed on promastigote forms because it is much easier to work with these forms in vitro. 


\begin{tabular}{|c|c|c|c|c|c|}
\hline \multirow[b]{2}{*}{ Compound } & \multicolumn{2}{|c|}{ IC50 (pM) } & \multirow{2}{*}{$\begin{array}{c}\text { Toxicity } \\
\text { J774_2 } \\
\text { macrophages } \\
I_{50}(\mu M)^{a} \\
\end{array}$} & \multicolumn{2}{|c|}{ SI b } \\
\hline & L. infantum & L. braziliensis & & L. infantum & L. braziliensis \\
\hline Glucantime & 18.0 & 25.6 & 15.2 & 1 & 1 \\
\hline Hmtpo & 63.4 & 60.6 & 99.8 & 1.6 & 1.6 \\
\hline 1 & 20.0 & 22.1 & 723.8 & 36 & 33 \\
\hline 2 & 24.4 & 31.5 & 945.5 & 39 & 30 \\
\hline 3 & 29.0 & 23.5 & 843.3 & 29 & 36 \\
\hline
\end{tabular}

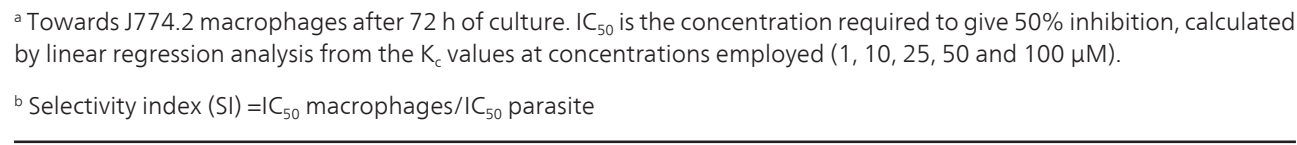

Table 2. In vitro activity of reference drugs, free $\mathrm{HmtpO}$ derivative and metal compounds 1, 2 and 3 against promastigote forms of Leishmania spp.

However, in our studies we also include the effects of these compounds on the forms that develop in the host (amastigotes). This study is of great importance to determine effects in the definitive host and thus it gives a better idea of the potential application as antiparasitic drugs.

To predict the effect of metal complexes 1-3 on the capacity for infection and growth inhibition of intracellular forms of L. infantum and L. braziliensis, adherent J774.2 macrophages $\left(1 \times 10^{5}\right.$ macrophages) were incubated for two days and then infected with $1 \times 10^{6}$ promastigote forms of L. infantum and L. braziliensis for $12 \mathrm{~h}$. Non-phagocytosed parasites were afterwards removed and culture was kept in fresh medium for 10 days. Parasites invaded cells and then converted into amastigotes within one day after infection. On the $10^{\text {th }}$ day, the rate of host-cell infection reached the maximum. When drugs 1-3 were added at their respective $\mathrm{IC}_{25}$ concentration to macrophages infected with Leishmania spp. promastigote forms in exponential growth phase, infection rate decreased significantly after $12 \mathrm{~h}$ with respect to control measurements, following the trend $1>3>2$ for L. infantum and $3>1>2$ for L. braziliensis, with percentages of infestationinhibition capacity of $84 \%, 79 \%$ and $67 \%$, respectively, in the case of L. infantum and $86 \%, 79 \%$ and $75 \%$, respectively, in the case of L. braziliensis. These values are remarkably higher than those for inhibition by Glucantime $(56 \%$ and $36 \%$ for L. infantum and L. braziliensis, respectively). The three complexes inhibited Leishmania spp. amastigote replication in macrophage cells in vitro, following a similar pattern to that for infection rate inhibition and again being more effective than reference drug. Although not always it is possible to establish a direct relationship between drug action on extracelular promastigote and intracellular amastigote forms, in case of compound 3, it was effective against both forms.

\subsection{Studies on the mechanism of action}

In order to investigate the possible mechanism of action of metal compounds 1-3 on the parasite, their effect on metabolite excretion is analyzed, and microscopy studies on the treated 
parasites are carried out to visualize any ultrastructural alteration that may be provocked by the compounds.

\subsubsection{Metabolite excretion effect}

To the best of our knowledge, none of the trypanosomatids studied is capable of completely degrading glucose to $\mathrm{CO}_{2}$ under aerobic conditions, so they excret a great part of the carbon skeleton into medium as fermented metabolites, which can differ according to the employed species.[46] Leishmania spp. have a high rate of glucose consumption, thereby acidifying culture medium due to incomplete oxidation to acids. ${ }^{1} \mathrm{H}-\mathrm{NMR}$ spectra enable us to determine fermented metabolites that are excreted by the parasites during their in vitro culture. One of the major metabolites excreted by Leishmania spp. is succinate, the main role of which is probably to maintain the glycosomal redox balance by providing two glycosomal oxidoreductase enzymes. These enzymes allow reoxidation of NADH that is produced by glyceraldehyde-3-phosphate dehydrogenase in the glycolytic pathway. Succinic fermentation offers one significant advantage, since it requires only half of the produced phosphoenolpyruvate (PEP) to maintain the $\mathrm{NAD}^{+} / \mathrm{NADH}$ balance. The remaining PEP is converted into acetate, depending on the species being considered. Figure 10 (on the left) shows ${ }^{1} \mathrm{H}-\mathrm{NMR}$ spectrum of cell-free medium four days after inoculation with L. infantum. Additional peaks, corresponding to the major metabolites that were produced and excreted during growth, could be detected when this spectrum was compared with the one made in fresh medium. Taking into account that $L$. infantum excretes mainly succinate and acetate, ${ }^{1} \mathrm{H}-\mathrm{NMR}$ spectra show that only compound 2 significantly altered excreted metabolites by L. infantum. When promastigote forms of $L$. infantum were treated with compound 2 at $\mathrm{IC}_{25}$ doses, the excretion of catabolites (succinate and acetate) was clearly disturbed and a new peak, identified as pyruvate, appeared (Figure 10). These results mean that compound 2 inhibits glycosomal enzymes, causing pyruvate to be excreted as a final metabolite. On the other hand, compounds 1 and 3 inhibite excreted metabolites only slightly. In the case of L. braziliensis, compounds 1-3 showed a similar behavior as for L. infantum, being again compound 2 the most inhibitory.

\subsubsection{Ultrastructural alterations}

Transmission electron microscopy images showed that compounds 1-3 induced morphological alterations in L. infantum and L. braziliensis promastigotes when parasites were treated with the respective $\mathrm{IC}_{25}$. Compound 2 was the most effective against both parasite species. 


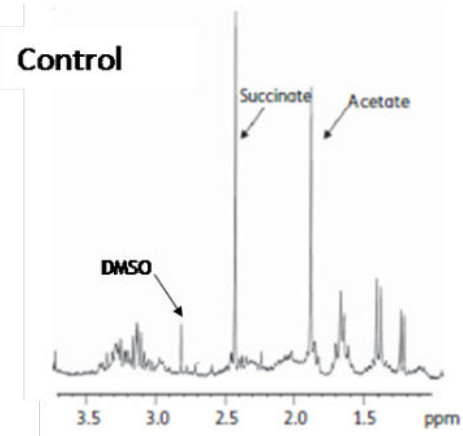

\section{Treated with 2}

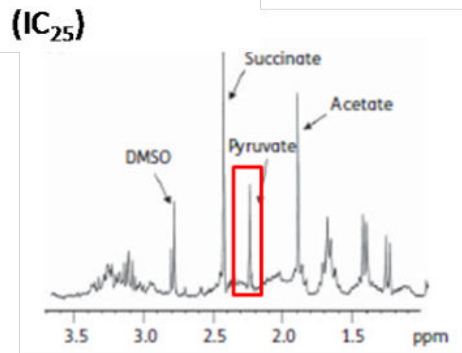

Figure 10. NMR spectra of promastigote forms of L. infantum, which show the characteristic peaks of the major excreted metabolites of non-treated parasites (left) and parasites that have been treated with $\mathrm{IC}_{25}$ of compound 2 (right) for four days. 


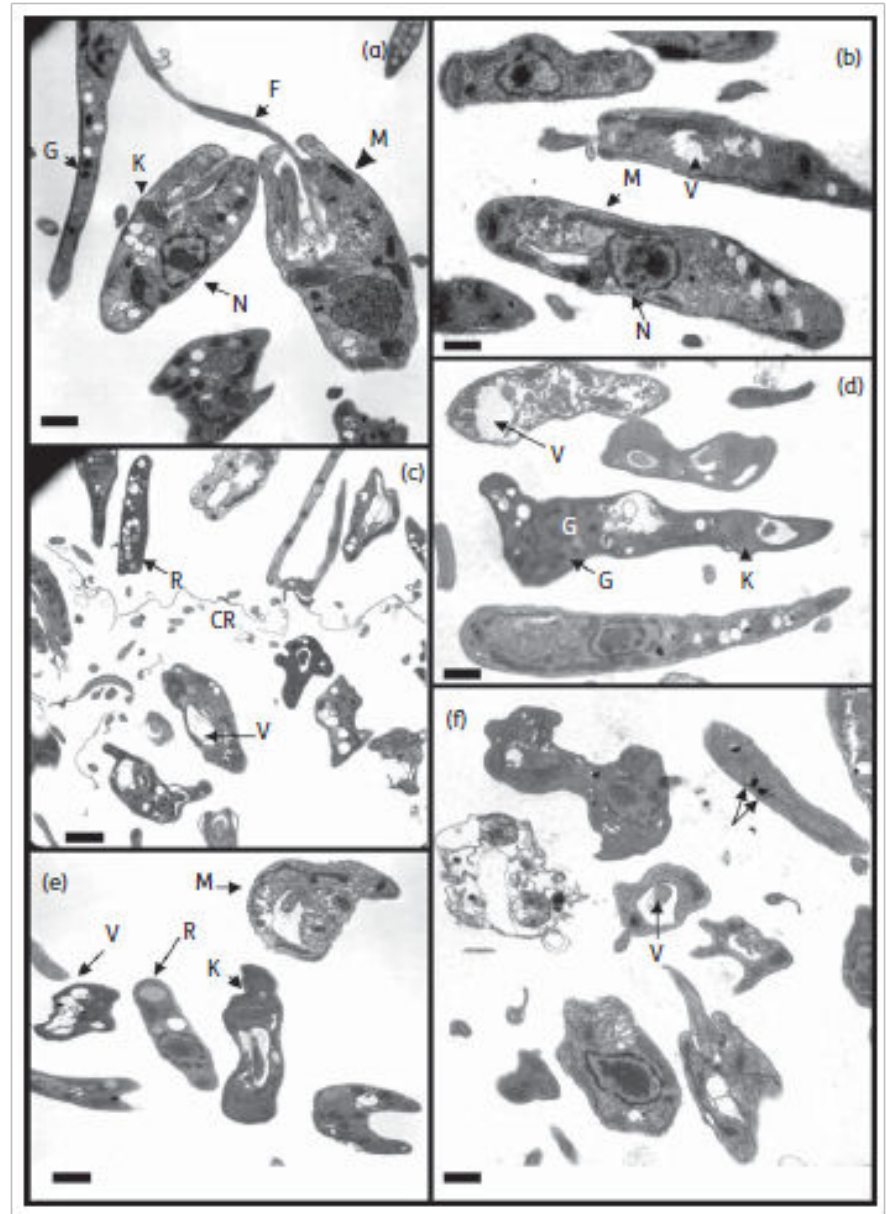

Figure 11. TEM images showing ultrastructural alterations in L. infantum and L. braziliensis after being treated with compounds 1, 2 and 3 (at $I C_{25}$ concentrations) for $72 \mathrm{~h}$. (a) Control parasite of L. infantum showing organelles with their characteristic aspect, such as nucleus $(\mathrm{N})$, kinetoplast $(\mathrm{K})$, flagellum (F), glycosomes $(\mathrm{G})$ and mitochondrion (M). Bar $=1.00 \mu \mathrm{m}$. (b) Control parasite of $L$. braziliensis with structures such as nucleus $(\mathrm{N})$, vacuoles $(\mathrm{V})$ and mitochondrion (M). Bar=1.00 $\mu \mathrm{m}$. (c) L. infantum treated with compound 2, showing cellular rest (CR), intense vacuolization (V) and reservosomes (R). Bar=1.59 $\mu \mathrm{m}$. (d) L. infantum treated with compound 3, showing electrodense cytoplasm, vacuoles $(V)$, glycosomes $(\mathrm{G})$ and kinetoplast $(\mathrm{K})$. Bar=1.00 $\mu \mathrm{m}$. (e) L. braziliensis treated with compound 1, showing intense vacuolization (V), giant reservosomes (R) and kinetoplast (K) and swelling mitochondrion (M). Bar=1.00 $\mu$ m. (f) Promastigotes of $L$. braziliensis treated with compound 2, with vacuoles $(V)$ and electrodense organelles (arrows). Bar=1.00 $\mu \mathrm{m}$.

After treating L. braziliensis promastigotes with compound 2, many of the parasites appeared dead and others adopted distorted shapes, while in others a uniformly electrodense cytoplasm was formed, in which no cytoplasmic organelles were visible. Parasites vacuolization was pronounced and many of these vacuoles contained strongly electrodense inclusions. In case of L. infantum, compound 2 led mostly to cell destruction (Figure 11c), which was evident from 
the presence of a great quantity of cell remains in supernatant. Likewise parasites had strongly electrodense cytoplasm with intense vacuolization, with both empty vacuoles and membranes, and reservosomes, which appeared in greater numbers than in non-treated promastigotes (Figure 11a).

On the other hand, compound 1 was again very effective against $L$. braziliensis as some parasites appeared dead and others completely altered (Figure 11e), replete with reservosomes and enormous vacuoles. Some promastigotes appeared to be distorted and strongly electrodense, and showed condensed kinetoplast and very swollen mitochondria. In contrast, compound 3 was effective against L. infantum (Figure 11d), whose alterations were similar to those already described, with unrecognizable parasites, filled with vacuoles, which distorted their morphology, as well as a great quantity of reservosomes that occupied practically the entire cytoplasm. In these parasites kinetoplast and mitochondria also appeared swollen, resulting in a strongly electrodense cytoplasm. Dead parasites were also visible.

\subsection{Final remarks}

In addition to these studies, it should be noted that compounds 1-3 have displayed a high in vitro activity against both extra and intracellular forms of T. cruzi and are effective at concentrations similar to those of benznidazole. At the same time, they are much less toxic for host cells than the latter. Moreover antileishmanial activity of metal compounds is much higher than that of isolated HmtpO ligand, which is an evidence of the critical role of metal ions in antiparasitic activity. Furthermore, promising in vivo activity was observed for all of them, with results consistent with those observed in vitro.

\section{Conclusion and future trends}

In comparison with economically more attractive diseases like cancer, cardio-vascular problems and allergies, commercial interest in developing new antiparasitics is still rather low. Low income of most of the people affected by leishmaniasis, as it is the case for other tropical diseases, discourages big pharmaceutical companies from investing in developing new therapies. Therefore there is an urgent need to investigate into new drugs with low cost of production but also with high efficacy and selectivity.

Research on metal-based compounds to treat leishmaniasis has resurged in the last years and significant progress has been made. The possibility to finely tune their reactivity through a change of the metal ion and appropiate choice of the ligand/s makes of metal compounds promising alternatives to fight this disease in a cost-effective way.

Optimization of currently available metal-based drugs such as antimonials through use of nanovehicles and attachment of targeting moieties may be an interesting option to overcome antimonials resistance problems and maybe the quickest way to produce effective results. Therapeutic effects might be enhanced by using e.g. metal nanoparticles as delivery carriers, which depending on the metal, might be able to produce high amounts of reactive oxygen species and induce oxidative stress to the parasites. 
On the other hand, significant advances in parasite genoma sequences and identification of targets in the last years along with an increasing understanding of metals interactions with a wide range of biomolecules, will contribute to development of highly efficient target-specific metal-based drugs in the future while avoiding recurring to time-consuming drug screening methodologies.

Meanwhile some authors have pointed at the metal-drug synergism approach as a very useful alternative for drug design at the moment.

\section{Author details}

Ana B. Caballero ${ }^{1}$, Juan M. Salas² and Manuel Sánchez-Moreno ${ }^{3}$

1 School of Chemistry, University of Birmingham, Birmingham, United Kingdom

2 Department of Inorganic Chemistry, School of Sciences, University of Granada, Granada, Spain

3 Department of Parasitology, School of Sciences, University of Granada, Granada, Spain

\section{References}

[1] Singh MP, Mishra M, Khan AB, Ramdas SL, Panjiyar S. Gold treatment for kala-azar. Br. Med. J. 1989; 2991318.

[2] Bruijnincx PC, Sadler PJ. New Trends for Metal Complexes with Anticancer Activity. Curr Opin Chem Biol. 2008; 12(2) 197-206.

[3] Sánchez-Delgado RA, Anzellotti A. Metal complexes as chemotherapeutic agents against tropical diseases: trypanosomiasis, malaria and leishmaniasis. Mini-Reviews in Medicinal Chemistry 2004; 423-30.

[4] Farrell NP. Transition Metal Complexes as Drugs and Chemotherapeutic Agents. In: R. Ugo, B. R. James (eds.) Catalysis by Metal Complexes. Vol 11. Dordrecht: Kluwer Academic Publishers; 1989. p. 222-242.

[5] Dubar F, Khalife J, Brocard J, Dive D, Biot C. Ferroquine, an Ingenious Antimalarial Drug -Thoughts on the Mechanism of Action. Molecules 2008; 13 2900-2907.

[6] Nogueira-Silva JJ, Pavanelli WR, Salazar Gutierrez FR, Chagas Alves Lima F, Borges Ferreira da Silva A, Santana Silva J, Wagner Franco D. Complexation of the anti-Trypanosoma cruzi Drug Benznidazole Improves Solubility and Efficacy. J. Med. Chem. $2008 ; 514104-4114$. 
[7] Navarro M, Gabbiani C, Messori L, Gambino D. Metal-based drugs for malaria, trypanosomiasis and leishmaniasis: recent achievements and perspectives. Drug Discovery Today 2010; 15 1070-1078.

[8] Das S, Roy P, Mondal S, Bera T, Mukherjee A. One pot synthesis of gold nanoparticles and application in chemotherapy of wild and resistant type visceral leishmaniasis. Colloids and Surfaces B: Biointerfaces 2013; 107 27-34.

[9] Beheshti N, Soflaei S, Shakibaie M, Yazdi MH, Ghaffairfar F, Dalimi A, Shahverdi AR. Efficacy of biogenic selenium nanoparticles against Leishmania major: In vitro and in vivo studies. Journal of Trace Elements in Medicine and Biology 2013; 27 203-207.

[10] Soflaei S, Dalimi A, Ghaffarifar F, Shakibaie M, Shahverdi AR, Shafiepour M. In Vitro Antiparasitic and Apoptotic Effects of Antimony Sulfide. Nanoparticles on Leishmania infantum. Journal of Parasitology Research 2012; doi 10.1155/2012/756568.

[11] Ouellette M, Drummelsmith J, Papadopoulou B. Leishmaniasis: drugs in the clinic, resistance and new developments. Drug Resistance Updates 2004; 7 257-266.

[12] Ashutosh, Sundar S, Goyal N. Molecular mechanisms of antimony resistance in Leishmania. Journal of Medical Microbiology 2007; 56 143-153.

[13] Baiocco P, Colotti G, Franceschini S, Ilari A. Molecular Basis of Antimony Treatment in Leishmaniasis. J. Med. Chem. 2009; 52 2603-2612.

[14] Sereno D, Holzmuller P, Mangot I, Cuny G, Ouaissi A, Lemesre J. Antimonial-mediated DNA fragmentation in Leishmania infantum amastigotes Antimicrob. Agents Chemother. 2001;45 2064-2069.

[15] Chakraborty AK, Majumder K. Mode of action of pentavalent antimonials: Specific inhibition of type I DNA topoisomerase of Leishmania donovani. Biochemical and Biophysical Research Communications 1988; 152(2) 605-611.

[16] Haldar AK, Sen P, Roy S. Use of Antimony in the Treatment of Leishmaniasis: Current Status and Future Directions. Molecular Biology International 2011, Article ID 571242. DOI: $10.4061 / 2011 / 571242$

[17] Berman, J.D.; Waddell, D.; Hanson, B.D. Biochemical mechanisms of the antileishmanial activity of sodium stibogluconate. Antimicrob. Agents Chemother. 1985, 27, 916-920.

[18] Sánchez-Delgado RA, Anzellotti A, Suárez L. Metal complexes as chemotherapeutic agents against tropical diseases: malaria, trypanosomiasis and leishmaniasis. In: Sigel A and Sigel H (eds). Metal Ions in Biological Systems Volume 41: Metal Ions and Their Complexes in Medication. FontisMedia and Marcel Dekker; 2004, p. 379-420.

[19] Martinez A, Carreon T, Iniguez E, Anzellotti A, Sanchez A, Tyan M, Sattler A, Herrera L, Maldonado RA, Sanchez-Delgado RA. Searching for New Chemotherapies for Tropical Diseases: Ruthenium-Clotrimazole Complexes Display High in Vitro Activ- 
ity against Leishmania major and Trypanosoma cruzi and Low Toxicity toward Normal Mammalian Cells. J Med Chem 2012; 55 3867-3877.

[20] Iniguez E, Sanchez A, Vasquez MA, Martınez A, Olivas J, Sattler A, Sanchez-Delgado RA, Maldonado RA. Metal-drug synergy: new ruthenium(II) complexes of ketoconazole are highly active against Leishmania major and Trypanosoma cruzi and nontoxic to human or murine normal cells. J Biol Inorg Chem 2013. DOI 10.1007/ s00775-013-1024-2

[21] Mesa-Valle CM, Moraleda-Lindez V, Craciunescu D, Osuna A. Antileishmanial Action of Organometallic Complexes of $\mathrm{Pt}(\mathrm{II})$ and $\mathrm{Rh}(\mathrm{I})$. Mem. Inst. Oswaldo Cruz, Rio de Janeiro 1996, 91, 625-633.

[22] Castilla JJ, Mesa-Valle MC, Sánchez-Moreno M, Arnedo T, Rosales MJ, Mascaro C, Craciunescu D, Osuna A. In vitro activity and biochemical effectiveness of new organometallic complexes of osmium(III) against Leishmania donovani and Trypanosoma cruzi. Arzneim.-Forsch.-Drug Res. 1996, 46, 990-996.

[23] Noleto GR, Mercê ALR, Iacomini M, Gorin PAJ, Soccol VT, Oliveira MBM. Effects of a lichen galactomannan and its vanadyl (IV) complex on peritoneal macrophages and leishmanicidal activity. Mol. Cell. Biochem. 2002; 233 73-83.

[24] Selzer PM, Pingel S, Hsieh I, Ugele B, Chan VJ, Engel JC, Bogyo M, Russell DG, Sakanari JA, McKerrow JH. Cysteine protease inhibitors as chemotherapy: Lessons from a parasite target. Proc. Natl. Acad. Sci. U. S. A. 1999; 96 11015-11022.

[25] Fricker SP. Cysteine proteases as targets for metal-based drugs. Metallomics 2010; 2 366-377.

[26] a) Kinnamon K, Steck EA, Rane ES. Activity of antitumor drugs against African trypanosomes. Antimicrob. Agents Chemother. 1979; 15 (2) 157-160. (b) Farrell NP, Williamson J, McLaren DJM. Trypanocidal and antitumour activity of platinum-metal and platinum-metal-drug dual-function complexes. Biochem. Pharmacol. 1984; 961-971.

[27] Navarro M, Cisneros-Fajardo EJ, Sierralta A, Fernández- Mestre M, Silva P, Arrieche D, Marchán E. Design of copper DNA intercalators with leishmanicidal activity. J. Biol. Inorg. Chem. 2003;8 401-408.

[28] Navarro M, Hernandez C, Colmenares I, Hernandez P, Fernandez M, Sierraalta A, Marchan E. Synthesis and characterization of $\left[\mathrm{Au}(\mathrm{dppz})_{2}\right] \mathrm{Cl}_{3}$. DNA interaction studies and biological activity against Leishmania (L) Mexicana. Journal of Inorganic Biochemistry. 2007; 101 111-116.

[29] Lowe G, Droz AS, Vilaivan T, Weaver GW, Tweedale L, Pratt JM, Rock P, Yardley V, Croft SL. Cytotoxicity of (2, 2':6', 2' -terpyridine) platinum (II) complexes to Leishmania donovani, Trypanosoma cruzi and Trypanosoma brucei. J. Med. Chem. 1999; 42, 9991006. 
[30] Gambino D. Potentiality of vanadium compounds as anti-parasitic agents.Coordination Chemistry Reviews 2011; 255 (19-20) 2193-2203.

[31] Benítez J, Becco L, Correia I, Milena Leal S, Guiset H, Costa Pessoa J, Lorenzo J, Aviles F, Escobar P, Moreno V, Garat B, Gambino D. Vanadium polypyridyl compounds as potential antiparasitic and antitumoral agents: new achievements. J. Inorg. Biochem. 2011; 105 303-312.

[32] Neldner KH, Hambidge KM, Walravens PA. Acrodermatitis enteropathica. Int J Dermatol. 1978;17(5) 380-387.

[33] Minodier P, Parola P. Cutaneous leishmaniasis treatment. Travel Medicine and Infectious Disease 2007; 5, 150-158.

[34] Yazdanpanah MJ, Banihashemi M, Pezeshkpoor F, Khajedaluee M, Famili S, Rodi IT, Yousefzadeh H. Comparison of Oral Zinc Sulfate with Systemic Meglumine Antimoniate in the Treatment of Cutaneous Leishmaniasis. Dermatology Research and Practice 2011, doi 10.1155/2011/269515.

[35] Al-Mulla Hummadi YM, Al-Bashir NM, Najim RA. The mechanism behind the antileishmanial effect of zinc sulphate. II. Effects on the enzymes of the parasites. Annals of Tropical Medicine and Parasitology 2005; 99(2) 131-139.

[36] Combs Jr GF. Selenium and cancer. In: Garewal HS, editor. Antioxidants and disease prevention. New York, NY: CRC Press; 1997. p. 97-113.

[37] Levander OA. Selenium and sulfur antioxidant protective systems, relationships with vitamin E and malaria. Proc Sot Exp Biol Med 1992; 200 255-259.

[38] Mukhopadhyay R, Madhubala R. Effect of antioxidants on the growth and polyamine levels of Leishmania donovani. BiochemicalPharmacology 1994; 47(4) 611-615.

[39] Fischer G. Recent Progress in 1, 2, 4-Triazolo[1, 5-a]pyrimidine Chemistry. Adv. Heterocycl. Chem. 2008; 95 143-219.

[40] Ramirez-Macias I, Marin C, Salas JM, Caballero A, Rosales MJ, Villegas N, Rodriguez-Dieguez A, Barea E, Sanchez-Moreno M. Biological activity of three novel complexes with the ligand 5-methyl-1, 2, 4-triazolo[1, 5-a]pyrimidin-7(4H)-one against Leishmania spp. J Antimicrob Chemother 2011; 66 813-819.

[41] Venier-Julienne MC, Vouldoukis I, Monjour L, Benoit JP. In vitro study of the antileishmanial activity of biodegradable nanoparticles.Journal of Drug Targeting 1995; 3(1) 23-29. (b) Durand R, Paul M, Rivollet D, Fessi H, Houin R, Astier A, Deniau M. Activity of pentamidine-loaded poly (D, L-lactide) nanoparticles against Leishmania infantum in a murine model, " Parasite 1997; 4(4) 331-336.

[42] Allahverdiyev AM, Abamor ES, Bagirova M, Ustundag CB, Kaya C, Kaya F, Rafailovich M. Antileishmanial effect of silver nanoparticles and their enhanced antiparasit- 
ic activity under ultraviolet light. International Journal of Nanomedicine 2011; 6 2705-2714.

[43] a) Salas JM, Romero MA, Sánchez MP, Quirós M. Metal complexes of [1, 2, 4]triazolo[1, 5-a]pyrimidine derivatives. Coord. Chem. Rev. 1999; 193-195 1119-1142. (b) Caballero AB, Maclaren JK, Rodríguez-Diéguez A, Vidal I, Dobado JA, Salas JM, Janiak C. Dinuclear silver(I) complexes for the design of metal-ligand networks based on triazolopyrimidines. Dalton Trans. 2011; 40(44) 11845-55; (c) Caballero AB, RodríguezDiéguez A, Barea E, Quirós M, Salas JM. Influence of pseudohalide ligands on the structural versatility and properties of novel ternary metal complexes with 1, 2, 4-triazolo[1, 5-a]pyrimidine. CrystEngComm 2010; 12 3038; (d) Abul Haj M, Quirós M, Salas JM, Dobado JA, Molina J, Basallote MG, Máñez MA. Structurally different dinuclear copper(II) complexes with the same triazolopyrimidine bridging ligand. Eur. J. Inorg. Chem. 2002; 811-818.

[44] Caballero AB, Rodriguez-Dieguez A, Lezama L, Barea E, Salas JM. Structural and magnetic properties of three novel complexes with the versatile ligand 5-methyl-1, 2, 4-triazolo[1, 5-a]pyrimidin-7(4H)-one. Dalton Transactions 2011; 40(19), 5180-5187.

[45] Tiuman TS, Ueda-Nakamura T, Garcia Cortez DA, Dias Filho BP, Morgado-Díaz JA, de Souza W, Nakamura CV. Antileishmanial activity of parthenolide, a sesquiterpene lactone isolated from Tanacetum parthenium. Antimicrob. Agents Chemother. 2005; 49 176-182.

[46] Bringaud F, Riviere L, Coustou V. Energy metabolism of trypanosomatids: adaptation to available carbon sources. Mol BiochemParasitol 2006; 149 1-9. 
